\title{
Corrigendum to "Inhibitory Effect of Paquinimod on a Murine Model of Neutrophilic Asthma Induced by Ovalbumin with Complete Freund's Adjuvant"
}

\author{
Jong-Uk Lee $\mathbb{D}^{1},{ }^{1}$ Jong Sook Park $\mathbb{D},{ }^{2}$ Ji Ae Jun $\mathbb{D}^{\circ},{ }^{3}$ Min Kyung Kim $\mathbb{D}^{1},{ }^{1}$ Hun Soo Chang $\mathbb{D}^{1}$ \\ Dong Gyu Baek $\left(\mathbb{D},{ }^{1}\right.$ Hyun Ji Song $\mathbb{1},{ }^{1}$ Myung-Sin Kim $\mathbb{1},{ }^{4}$, and Choon-Sik Park $\mathbb{1}^{2,3}$ \\ ${ }^{1}$ Department of Interdisciplinary Program in Biomedical Science Major, Soonchunhyang Graduate School, \\ Bucheon, Republic of Korea \\ ${ }^{2}$ Division of Allergy and Respiratory Medicine, Department of Internal Medicine, Soonchunhyang University Bucheon Hospital, \\ Bucheon, Republic of Korea \\ ${ }^{3}$ PulmoBioPark Co.,Ltd., Soonchunhyang University Bucheon Hospital, Bucheon, Republic of Korea \\ ${ }^{4}$ Department of Internal Medicine, Soonchunhyang University Gumi Hospital, Gumi, \\ Gyeongsangbuk-do 39371, Republic of Korea \\ Correspondence should be addressed to Choon-Sik Park; mdcspark@hanmail.net
}

Received 24 May 2021; Accepted 24 May 2021; Published 14 June 2021

Copyright (C) 2021 Jong-Uk Lee et al. This is an open access article distributed under the Creative Commons Attribution License, which permits unrestricted use, distribution, and reproduction in any medium, provided the original work is properly cited.

In the article titled "Inhibitory Effect of Paquinimod on a Murine Model of Neutrophilic Asthma Induced by Ovalbumin with Complete Freund's Adjuvant" [1], due to an error during the preparation of the manuscript, the incorrect authors' contributions statement and funding statement were included in the published article. The correct acknowledgments section should read as follows.

\section{Authors' Contributions}

Lee JU, Park CS, and Park JS conceptualised the study. Lee JU, JA Jun, and MK Kim curated the data. HJ Song performed formal analysis. Park JS was involved in funding acquisition. HS Chang was responsible for methodology. JA Jun, HJ Song, and DG Baek validated the data. JU Lee and MK Kim visualised the study. Lee JU, Park JS, and CS Park prepared the original draft. Lee JU and CS Park reviewed and edited the manuscript. Lee and Jong-Sook Park contributed equally to this work.

\section{Acknowledgments}

This study was supported by a grant from the National Research Foundation of Korea (NRF) funded by the
Ministry of Education (2017R1D1A1B04029365) and a research grant from Soonchunhyang University to JS Park.

\section{References}

[1] J.-U. Lee, J. S. Park, A. J. Ji et al., "Inhibitory Effect of Paquinimod on a Murine Model of Neutrophilic Asthma Induced by Ovalbumin with Complete Freund's Adjuvant," Canadian Respiratory Journal, vol. 2021, Article ID 8896108, 9 pages, 2021. 